\title{
Managing the aorta in patients with a PRKG1 mutation
}

\author{
Elizabeth L. Norton, MS, ${ }^{\mathrm{a}}$ David Gordon, MD, ${ }^{\mathrm{b}}$ and Bo Yang, MD, $\mathrm{PhD},{ }^{\mathrm{a}}$ Ann Arbor, Mich
}

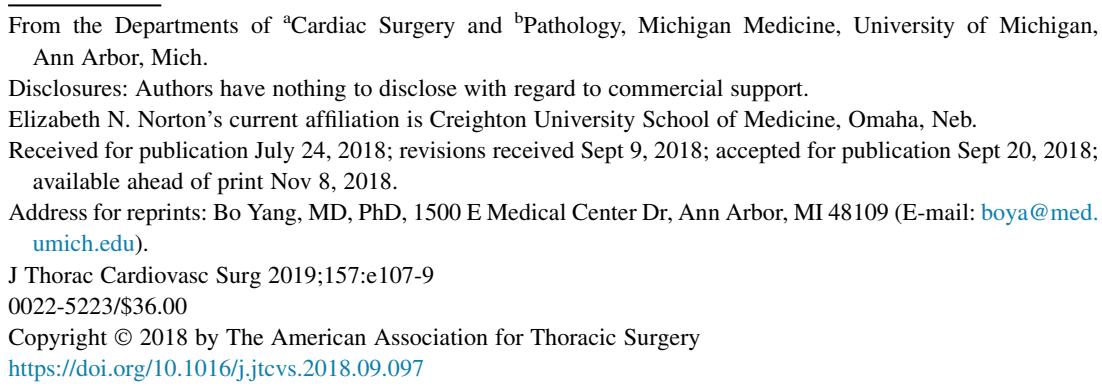

From the Departments of ${ }^{\mathrm{a} C a r d i a c}$ Surgery and ${ }^{\mathrm{b}}$ Pathology, Michigan Medicine, University of Michigan, Ann Arbor, Mich.

Disclosures: Authors have nothing to disclose with regard to commercial support.

Elizabeth N. Norton's current affiliation is Creighton University School of Medicine, Omaha, Neb.

Received for publication July 24, 2018; revisions received Sept 9, 2018; accepted for publication Sept 20, 2018; available ahead of print Nov 8, 2018.

Address for reprints: Bo Yang, MD, PhD, 1500 E Medical Center Dr, Ann Arbor, MI 48109 (E-mail: boya@med. umich.edu).

J Thorac Cardiovasc Surg 2019;157:e107-9

$0022-5223 / \$ 36.00$

Copyright $(2018$ by The American Association for Thoracic Surgery

https://doi.org/10.1016/j.jtcvs.2018.09.097

- Video clip is available online.

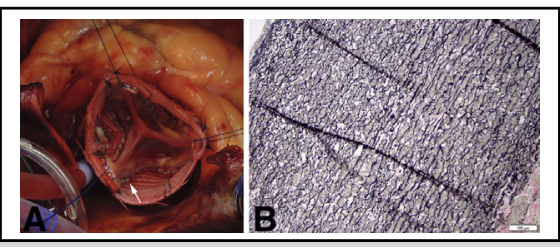

Extremely thin remnant of the aortic sinus wall and mild diffuse elastica fragmentation.

Central Message

Aggressive prophylactic replacement of the proximal aorta should be considered for patients with a pathogenic PRKGl mutation and family history of thoracic aortic dissection.

See Commentary page e111.
Around $25 \%$ of thoracic aortic aneurysms and dissections (TAADs) result from single gene mutations, ${ }^{1}$ including mutations in PRKG $1 .^{2,3}$ Current American Heart Association/American College of Cardiology guidelines provide recommendations for other mutations, in genes such as FBN1, TGFBR1, and $M Y H 11,4,5$ but not specifically PRKGl. We present a patient with a known PRKGl mutation and a story about her family (Video 1).

\section{CLINICAL SUMMARY}

A 47-year-old woman with a strong family history of TAAD was found to have a PRKGl heterozygous mutation. Her $P R K G 1$-positive brother experienced an aortic dissection at age 35 years, a niece died at age 16 years from an aortic dissection, 1 brother died at age 16 days from a congenital heart defect (ie, 3-chambered heart), another brother was stillborn at 7 months, among her 4 daughters, 2 are negative carriers and 2 are PRKG1 mutation-positive, and 1 granddaughter has the PRGK1 mutation. Both affected daughters (diagnosed at ages 24 and 22 years) were found to have tricuspid aortic valves, normal ejection fractions, and the older had a $29 \mathrm{~mm}$ aortic root whereas the younger had a 23 to $25 \mathrm{~mm}$ aortic root and $29 \mathrm{~mm}$ ascending aorta. The patient underwent an echocardiogram in her 30s when her brother experienced an aortic dissection, and all measurements were within normal ranges. Following positive genetic testing, an echocardiogram demonstrated a trileaflet aortic valve with trace aortic insufficiency and a left ventricular ejection fraction of $62 \%$. Computed tomography angiogram revealed a $37 \mathrm{~mm}$ aortic root and $38 \mathrm{~mm}$ ascending aorta (Figure 1).

The patient experienced significant anxiety regarding possible aortic dissection and eventually underwent a valve-sparing aortic root replacement (David V procedure-Stanford modification, using 32- and 24-mm Hemashield Dacron [Maquet, Rastatt, Germany] grafts) and ascending aorta replacement (as much as possible without circulatory arrest). The distal anastomosis was completed without surgical adjuncts (Figure 2, B). Most

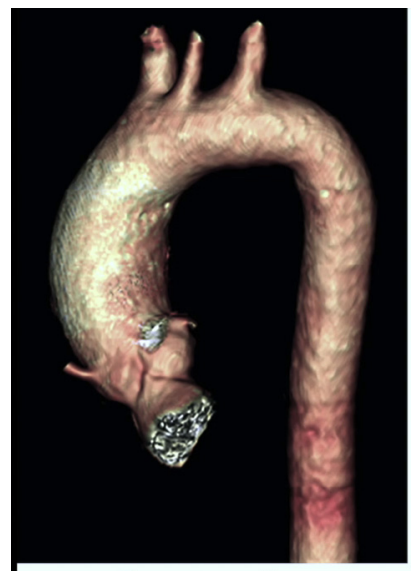

Preoperative

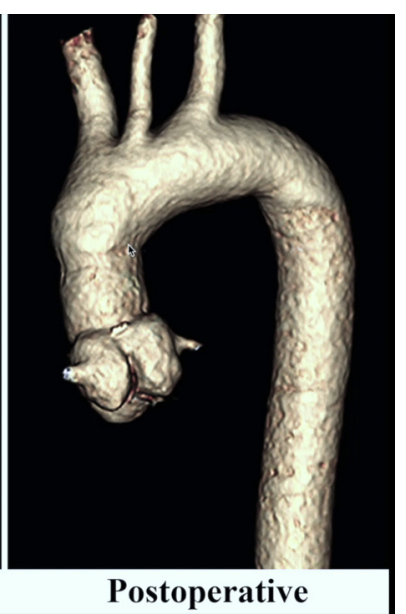

Postoperative
VIDEO 1. Discussion of prophylactic management of the aortic root and ascending aorta in patients with a known PRKG1 mutation. Video available at: https://www.jtcvs.org/article/S0022-5223(18)32605-9/fulltext. 


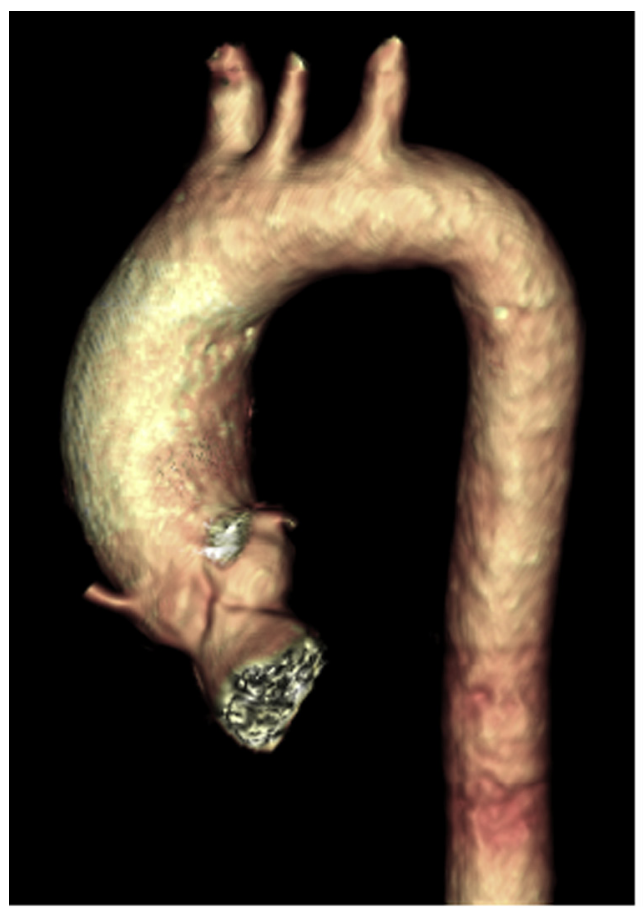

Preoperative

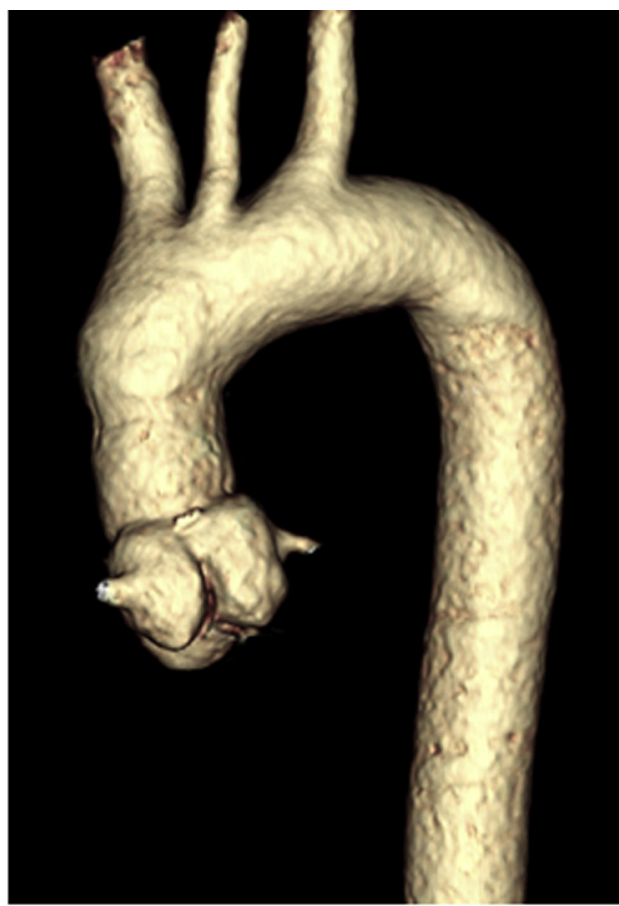

Postoperative

FIGURE 1. Computed tomography angiography scans before (left) and 3 months after (right) valve-sparing aortic root and ascending aorta replacement in a patient with a PRKGl mutation.

notably, the wall of the proximal ascending aorta and aortic root was exceptionally thin $(1 \mathrm{~mm})$, almost as thin as the outer layer of a dissected aorta (Figure 2, A). The right ventricular outflow tract could be visualized through the right coronary sinus wall. The distal ascending aortic wall was about 2-mm thick. The aorta was very fragile, and likely to tear during cannulation of the aortic arch. We used a single 3-0 Ethibond Exel purse-string suture (Ethicon, Bridgewater, NJ) without pledgets for aortic cannulation at the midarch and the cannulation site was reinforced with 50 prolene sutures in a figure- 8 fashion after decannulation. Histology showed thin aortic media without typical changes seen in patients with Marfan syndrome or Loeys-Dietz syndrome (Figure 2, $C$ and $D$ ). She has been doing well for $>1$ year with a competent aortic valve since her operation (Figure 1). On our patient's postoperative day 4, a 30year-old cousin with the same PRKGl mutation experienced an acute type A aortic dissection with mesenteric malperfusion and necrotic bowel.

\section{DISCUSSION}

Although mutation in PRKGl has been linked to familial $\mathrm{TAAD},{ }^{2,3}$ there is no evidence to guide the clinical management of affected patients, so clinical decision making is difficult. PRKGl encodes a type 1 cGMPdependent kinase involved in smooth muscle relaxation and contraction ${ }^{2,3}$ crucial in maintaining the integrity of the thoracic aortic wall throughout life. ${ }^{2}$ Patients with a PRKG1 mutation present with aortic dissection relatively early in life, around age 31 years (range, 17-51 years; $\mathrm{n}=31){ }^{2}$ In a study by Guo and colleagues, ${ }^{2}$ an acute type A aortic dissection occurred at an aortic root diameter of $37 \mathrm{~mm}$ and ascending aorta diameter of $43 \mathrm{~mm}$ in a 27 -year-old female patient. Gago-Diaz and colleagues ${ }^{3}$ reported on a female patient who experienced a type B dissection at age 41 years and a type $\mathrm{A}$ dissection at age 43 years. Before dissection, the aortic root measured $44 \mathrm{~mm}$ and the ascending aorta measured $37 \mathrm{~mm}$. Patients with the PRKG1 mutation are predisposed to an aggressive form of thoracic aortic disease, experiencing aortic events at relatively young ages and small aortic diameters., ${ }^{2,3}$ Therefore, prophylactic aortic repair should be considered at smaller aortic diameters in affected patients, but specific indications for prophylactic repair remain undecided. Although mutations in PRKGl are not the most common cause of genetic TAAD, guidelines regarding this specific presentation are warranted.

In the case presented, the intraoperative finding of an extremely thin aortic wall and strong family history of aortic dissections and death support early aggressive management of the aorta in patients with pathogenic PRKG1 variants, and if possible, valve-sparing aortic root replacement should be performed. The patient's affected daughters (now aged 26 and 24 years with normal aortic root and 

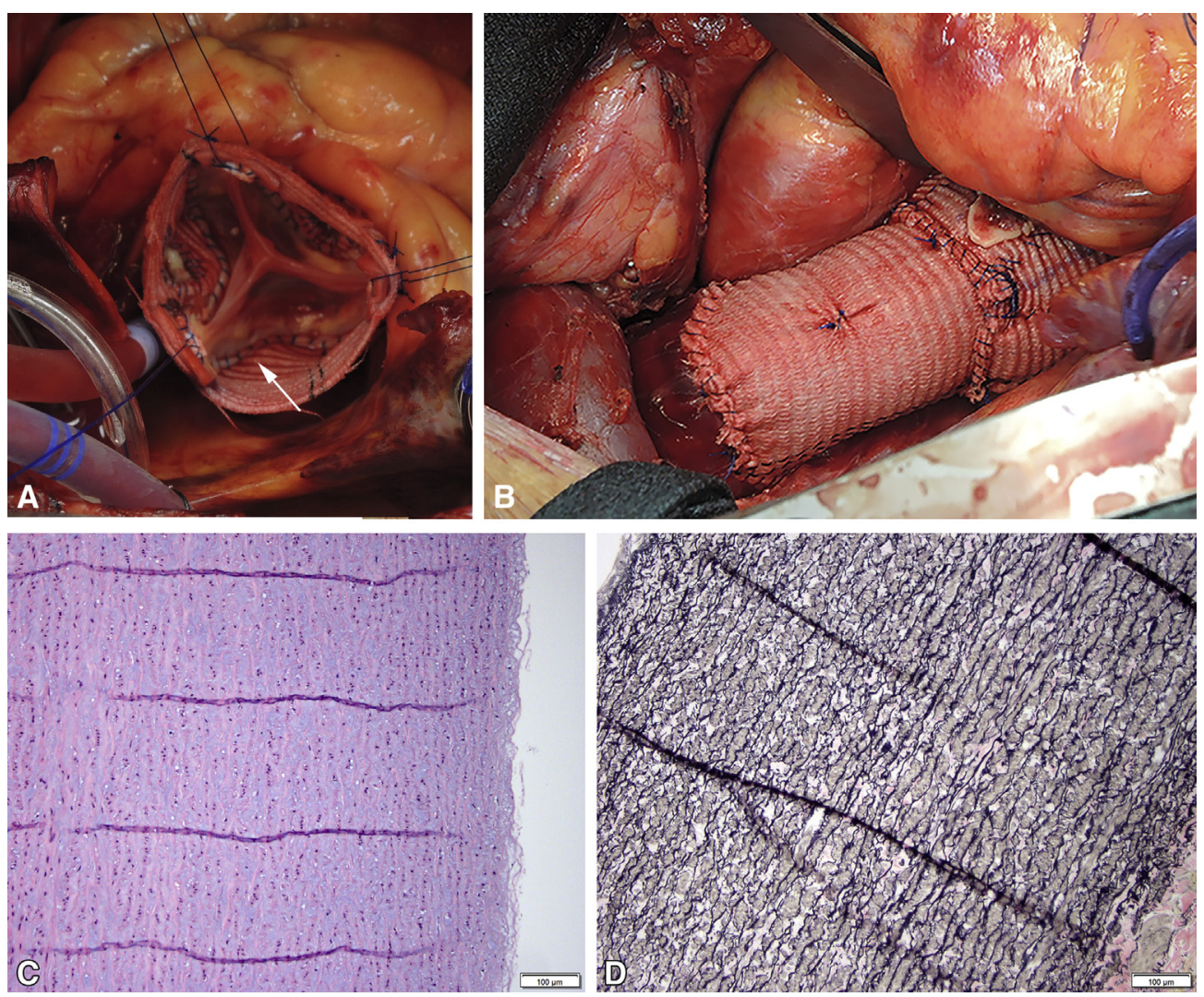

FIGURE 2. A, Valve-sparing root replacement (David procedure) in a patient with PRKG1 mutation and a thin and fragile aortic wall. Suture for aortic valve implantation was meticulously placed through the aortic annulus, which was strong enough to hold the suture. The arrow indicates the extremely thin remnant of the aortic sinus wall. B, Completion of the repair showing the distal anastomosis of the Hemashield Dacron graft to the ascending aorta. C, Moderate, multifocal intralamellar mucoid extracellular matrix accumulation without altering the arrangement of the lamellar units. Medial fibrosis and inflammation were also absent. Thus, overall the media displayed mild-to-moderate medial degeneration. Additionally, there was no evidence of aortitis or dissection (hematoxylin and eosin stain). D, The aortic media also displayed moderate, multifocal elastica fragmentation plus multifocal mild-tomoderate elastic fiber thinning. There was no elastic fiber disorganization, no smooth muscle loss or disorganization, and no laminar medial collapse (elastic Van Gieson stain).

ascending aorta dimensions) continue to be monitored with annual echocardiograms, but the question of when to consider prophylactic aortic repair remains.

Funding Source: Dr. Yang is supported by the NHLBI of NIH K08HL130614, R01HL141891, the Phil Jenkins Fund, David Hamilton Fund, and Darlene and Stephen J. Szatmari Fund.

\section{References}

1. Milewicz D, Hostetler E, Wallace S, Mellor-Crummey L, Gong L, Pannu H, et al. Precision medical and surgical management for thoracic aortic aneurysms and acute aortic dissections based on the causative mutant gene. J Cardiovasc Surg (Torino). 2016;57:172-7.

2. Guo DC, Regalado E, Casteel DE, Santos-Cortez RL, Gong L, Kim JJ, et al Recurrent gain-of-function mutation in PRKG1 causes thoracic aortic aneurysms and acute aortic dissections. Am J Hum Genet. 2013;93:398-404.

3. Gago-Diaz M, Blanco-Verea A, Teixido G, Huguet F, Gut M, Laurie S, et al PRKG1 and genetic diagnosis of early-onset thoracic aortic disease. Eur J Clin Invest. 2016;46:787-94.

4. Hiratzka LF, Bakris GL, Beckman JA, Bersin RM, Carr VF, Casey DE Jr, et al 2010 ACCF/AHA/AATS/ACR/ASA/SCA/SCAI/SIR/STS/SVM guidelines for the diagnosis and management of patients with thoracic aortic disease. Circulation. 2010;121:e266-369.

5. Mokashi SA, Svensson LG. Guidelines for the management of thoracic aortic disease in 2017. Gen Thorac Cardiovasc Surg. 2019;67:59-65. 